\title{
Primary Intimal Sarcoma of the Pulmonary Vein with Extension into the Left Atrium: A Case Report
}

\author{
Constantine D. Mavroudis ${ }^{1}$, Alfred Casillan ${ }^{1}$, Mark Rabbat ${ }^{2}$, Aileen Go ${ }^{3}$, Edward Melian ${ }^{4}$, \\ Mamdouh Bakhos $^{1}$, Christopher H. Wigfield ${ }^{{ }^{*}}$ \\ ${ }^{1}$ Department of Thoracic and Cardiovascular Surgery, Stritch School of Medicine, Loyola University Chicago, Maywood, USA; \\ ${ }^{2}$ Department of Cardiology, Stritch School of Medicine, Loyola University Chicago, Maywood, USA; ${ }^{3}$ Department of Oncology, \\ Stritch School of Medicine, Loyola University Chicago, Maywood, USA; ${ }^{4}$ Department of Radiation Oncology, Stritch School of \\ Medicine, Loyola University Chicago, Maywood, USA. \\ Email: ${ }^{*}$ cwigfield@lumc.edu
}

Received February $16^{\text {th }}, 2012$; revised March $19^{\text {th }}$, 2012; accepted March $25^{\text {th }}, 2012$

\begin{abstract}
Sarcoma originating from the pulmonary veins represents one of the rarest subtypes of sarcoma. We report the case of a 52-year-old woman presenting with cough and scant hemoptysis and ultimately diagnosed with a primary intimal sarcoma of the left inferior pulmonary vein extending into the left lower lobe of the lung. To our knowledge, this is the first reported case of such pathology in the literature. We present the case and critically appraise the literature for sarcomas of the great vessels.
\end{abstract}

Keywords: Sarcoma; Pulmonary Vein

\section{Case Report}

A 52-year-old female presented to her primary care physician complaining of a productive cough and intermittent streaks of hemoptysis. Her only significant medical history was asthma, which had been well-controlled for the majority of her adult life, and mild aortic insufficiency that had been discovered incidentally in a workup for palpitations over a decade earlier. She had no previous episodes of hemoptysis, and denied chest pain, dyspnea, fevers or chills and weight loss. There had been no traumatic injury to her chest. After her initial assessment by a primary care physician, she was prescribed antibiotics for a presumed pneumonia.

Her symptoms progressed despite oral antibiotics and a CT angiogram of the chest was obtained to evaluate for pulmonary embolism and to better visualize the lungs. The study demonstrated a large filling defect in the left inferior pulmonary vein with extension into the left atrium (Figure 1(a)). Three discrete lesions were also noted in the lower lobe of the left lung and there was a small left pleural effusion. No other lesions were noted on CT. She was then transferred to our institution for further evaluation.

On assessment, the patient had no respiratory compromise and was hemodynamically stable. Her physical

"Corresponding author. exam, including detailed respiratory and cardiovascular assessment, was noncontributory. Her basic laboratory findings were unremarkable; including a hemoglobin $13.8 \mathrm{~g} / \mathrm{dL}$, white blood cell count $9600 / \mathrm{mm}^{3}$, PTT $37.7 \mathrm{~s}$, PT 11.2 s, INR 1.1 and d-Dimer $580 \mu \mathrm{g} / \mathrm{L}$.

A heparin infusion had been commenced at outside hospital, sputum cultures were obtained, broad-spectrum antibiotics were started, and the patient was admitted to critical care for observation. Bronchoscopy was then performed to investigate the source of the patient's hemoptysis. No specific focal endobronchial lesion was identified. A left lower lobar subsegmental origin of minor bleeding was confirmed. Cytology from bronchial brushing was negative for malignancy. A transesophageal echocardiogram was also performed under the same general anaesthesia and demonstrated a dilated left atrium, with a heterogenous, sessile, mass, measuring $3.5 \times 2 \mathrm{~cm}$, which did not obstruct the mitral valve or the left ventricular outflow tract. Adequate pulmonary venous flow was demonstrated from the left upper pulmonary vein, but the left lower pulmonary vein could not be visualized. Aside from the known, mild aortic insufficiency, no other structural abnormalities were demonstrated. A cardiac MRI subsequently confirmed a large $3.1 \times 1.5 \times 2.8$ $\mathrm{cm}$ mass protruding into the left atrium and extending from the left inferior pulmonary vein (Figures 1(b) and 1(c)). No other lesions were found in cardiac MR. 
After discussing the results and differential diagnosis with the patient, informed consent was obtained for the excision of the atrial mass. A median sternotomy was performed and cardiopulmonary bypass was established. The left atrium was opened via the interatrial groove and a large mass was noted adherent to the posterior wall of the left atrium. The firm tumor appeared to originate from the left inferior pulmonary vein and completely obliterated the vascular lumen. The tumor had no interaction with mitral valve structures and no valvular intervention was required.

The posterior wall of the left atrium was resected along with the mass extending to the left inferior pulmonary vein. Frozen-section pathology described presence of a spindle cell tumor without further differentiation (Figure 2(a)). The posterior-lateral defect of the left atrium was repaired with a extra-cellular matrix patch excluding the left lower pulmonary vein return (CorMatrix Cardiovascular, Atlanta, GA, USA). After closure of the left atrium, a left lower lobectomy was performed to complete the resection of the mass (Figure 1(d)).

The patient's postoperative course and recovery were

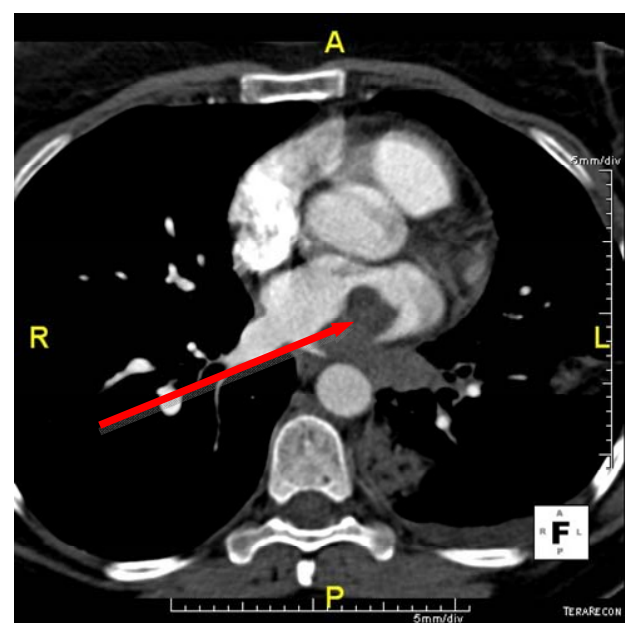

(a)

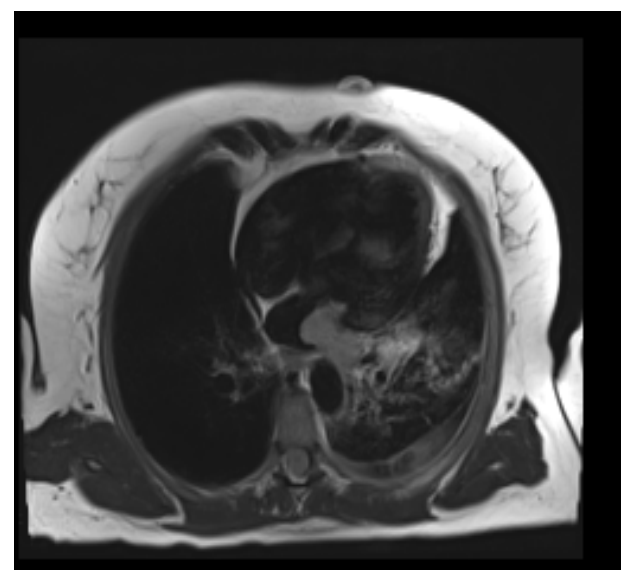

(b)

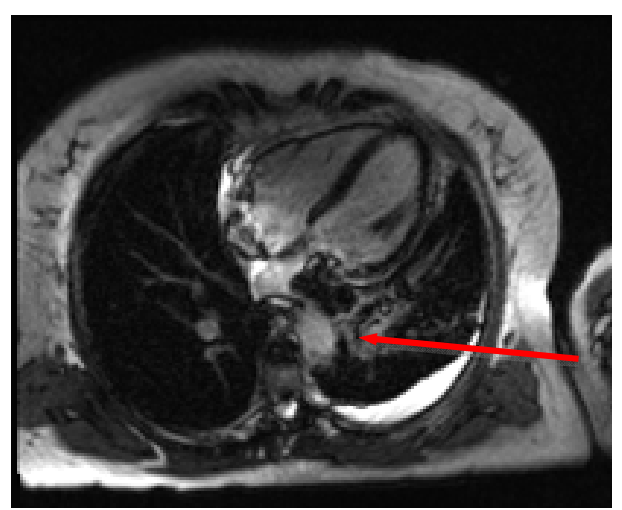

(c)

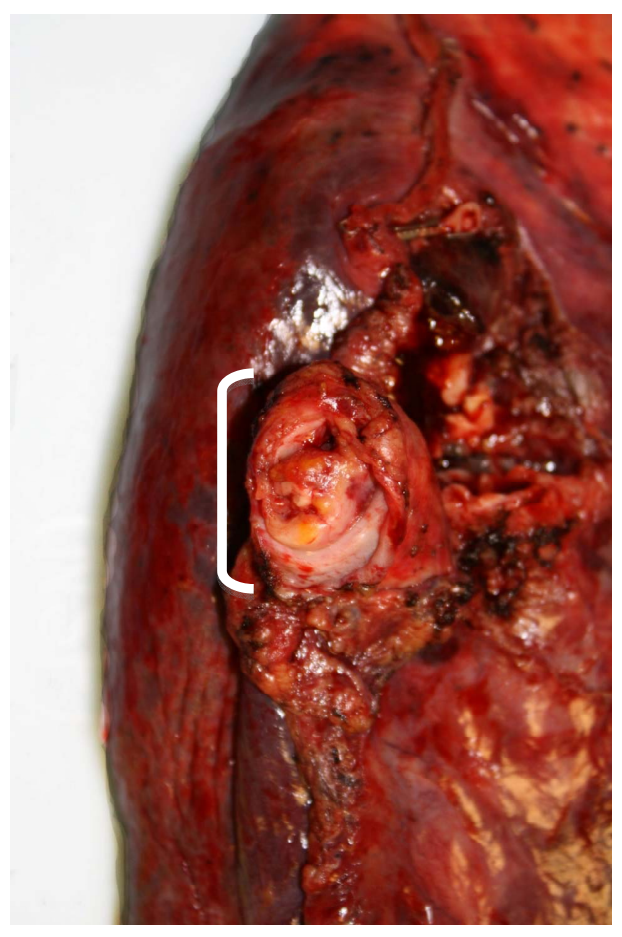

(d)

Figure 1. (a) CT scan demonstrating a large filling defect involving the left inferior pulmonary vein extending into the left atrium (arrow). Opacities in the left lower lobe and a left pleural effusion are present; (b) Four chamber dark blood imaging demonstrating the invasion of the mass into the left lower lobe of the lung; (c) Four chamber late gadolinium enhancement demonstrating patchy irregular enhancement of the mass consistent with a vascular tumor (arrow); (d) Left lower lung lobectomy specimen with 2.7 cm tumor protruding from the wall of the left inferior pulmonary vein. Bracket indicates $2.7 \mathrm{~cm}$ length of protruding mass.

uneventful. A repeat cardiac MRI was performed and confirmed complete resection of the left atrial and left lower lobe mass without residual tumor. Final pathologic evaluation revealed an adult-type, high grade, primary intimal sarcoma (fibrosarcoma) of the great vessels arising from the pulmonary veins with extension into the left 


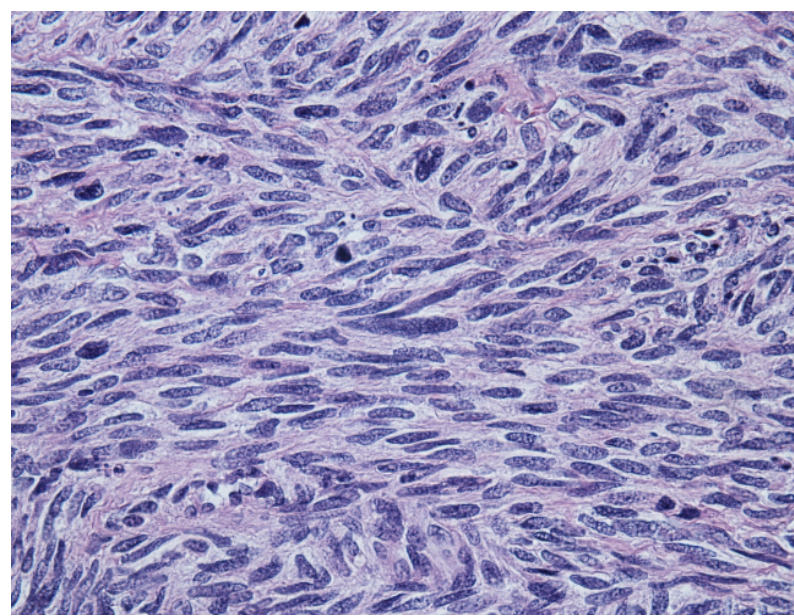

(a)

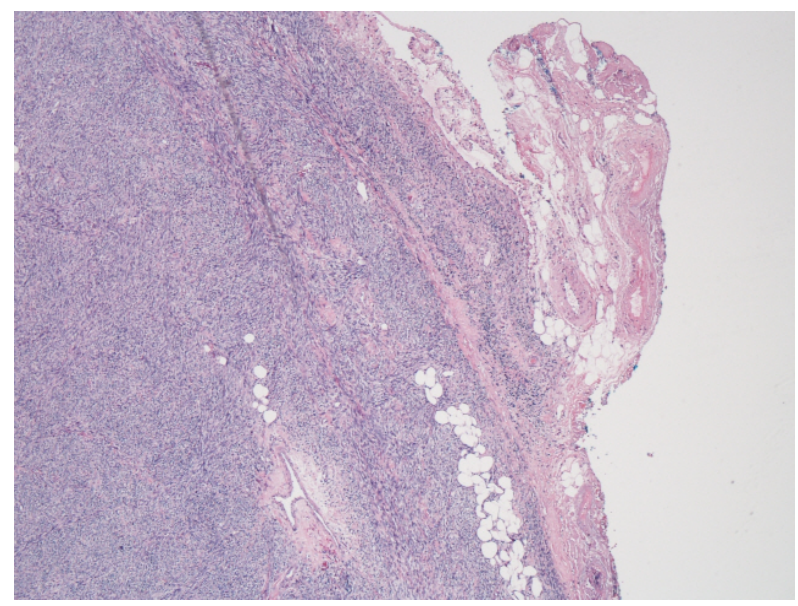

(b)

Figure 2. (a) Microscopic section showing tumor comprised of atypical spindled cells arranged in short fasicles with a herringbone pattern. Greater than 75 mitosis/hpf were identified (H + E × 25); (b) Microscopic section showing sarcoma infiltrating through the adventitia of the pulmonary and into peri-adventitial soft tissue $(H+E \times 1.26)$.

atrium and left lower lung. Lobectomy specimen confirmed regional extension but did not demonstrate evidence of infiltration into adjacent bronchus or lung parenchyma. Microscopic pulmonary vein margins were positive for sarcoma and the tumor was noted to extend through the pulmonary vein adventitia into the peri-adventitial tissue (Figure 2(b)). None of the five hilar and mediastinal lymph nodes (N1 and N2) examined had evidence of metastatic disease.

The patient underwent 45 Gy of radiotherapy in 25 fractions and an additional 14.4 Gy boost in 8 fractions using 3D conformal technique over 48 days, but opted not to pursue adjuvant chemotherapy after discussion of benefits and risks of systemic treatment. She remains without evidence of metastatic disease six months after the resection was completed and is being followed with serial imaging.

\section{Discussion}

Fewer than 400 cases of primary tumors of the great vessels have been reported in the literature [1]. Of these, fewer than 30 represent primary tumors of the pulmonary veins [2]. The vast majority of these tumors were leiomyosarcoma, with a single case reported as myxosarcoma [3] and another identified as myxoidfibrosarcoma [4]. The nonspecific signs and symptoms demonstrated by our patient are similar to those of leiomyosarcoma patients with involvement of the pulmonary vein. The differential diagnosis of such lesions requires detailed work up and specific imaging including TEE and cardiac MRI [5]. Pathology specimens obtained should be reviewed by experts in the field, since determining the exact origin of sarcomas associated with vascular structures can be complex.

To our knowledge, this is the first reported case of non-myxoid intimal sarcoma (fibrosarcoma) of the pulmonary vein. The most extensive series of patients with primary sarcoma of the pulmonary veins contained 17 cases with leiomyosarcoma [2]. Despite the difference in specific pathology, there were several noteworthy demographic and prognostic factors revealed in this analysis that are pertinent to our patient's presentation. There is a female predominance in leiomyosarcoma of the great vessels, most notably a 4.8:1 ratio found in leiomyosarcoma of the IVC [6]. The median age of presentation in cases of leiomyosarcoma of the pulmonary veins is 48 years. The most common presenting symptoms are dyspnea, hemoptysis, cough, chest pain, pleural effusion, and chest pain in decreasing order of frequency. The most common originating sites described for these tumors are in the right superior and left inferior pulmonary veins [2]. Our patient presented with typical demographics and presenting symptoms as the somewhat less rare leiomyosarcoma of the pulmonary veins.

The nonspecific signs and symptoms of both leiomyosarcoma and fibrosarcoma of the pulmonary veins make differential diagnostic efforts complex and diagnosis may be delayed. In the largest series of leiomyosarcoma of the pulmonary veins, roughly $50 \%$ of cases were diagnosed with CT and/or MRI angiography [2]. It is noteworthy that the tumor masses were thought to originate from the left atrium in the majority of these cases [2]. Bronchoscopy and Transesophageal echocardiography in conjunction with CT and cardiac MRI were required in rendering the likely diagnosis and directing urgent management in this case. The regional involvement of the left lower lobe and the inferior pulmonary vein origin, could not be demonstrated initially with bronchoscopy and transesophageal echocardiography. 
Diagnostic difficulties, however, are only one aspect of the poor overall prognosis of primary tumors of the pulmonary veins, as it is uniformly poor regardless of pathologic subtype. There are only two reported cases of patients surviving three or more years after treatment for leiomyosarcoma, and only a single report of a patient surviving longer than nine months after treatment for myxosarcoma [2,3]. Both of the leiomyosarcoma patients had undergone surgery with apparently complete resection and clear margins [2]. Following resection, there is still a high incidence of relapse, which underlines the need for effective neoadjuvant or adjuvant treatments [7]. In the treatment of resectable sarcomas, the use of adjuvant chemotherapy is still much debated [6,8,9]. An updated metananalysis of adjuvant chemotherapy did not demonstrate an overall survival advantage, although progression free survival was improved after Doxorubicin based chemotherapies [7]. It is reasonable to pursue a neoadjuvant approach depending on the histological subtype when local resection is not feasible although data is also lacking in this regard [10]. As this disease process is a rare entity, it is highly unlikely that a clinical trial with sufficient sample size would be done to determine the standard approach to treatment. Most of the information on primary cardiac and sarcomas of the great vessels are obtained from case reports and small retrospective studies. Complete resection seems to offer the best prognosis and chance for cure in these patients.

\section{REFERENCES}

[1] A. P. Burke and R. Virmani, "Tumors of the Great Vessels,” In: A. P. Burke and R. Virmani, Eds., Atlas of Tumor Pathology, Tumors of the Heart and Great Vessels, Armed Forces Institute of Pathology, Washington DC, 1996, pp. 211-226.

[2] T. Okuno, K. Matsuda, K. Ueyama, N. Oota, Y. Terada, Y. Hohjoh, T. Sakurai, T. Nakayama, M. Kitaichi and H. Yamabe, "Leiomyosarcoma of the Pulmonary Vein," $\mathrm{Pa}$ - thology International, Vol. 50, No. 10, 2000, pp. 839-846. doi:10.1046/j.1440-1827.2000.01116.x

[3] C. F. Hsuan, W. K. Tseng, C. H. Yang, T. L. Lee, P. Y. $\mathrm{Hu}$ and $\mathrm{C}$. C. Wu, "Primary Myxosarcoma of the Right Inferior Pulmonary Vein Presenting Clinically as Benign Left Atrial Myxoma with a Concurrent Right Lower Lung Tumor,” Circulation Journal, Vol. 73, No. 8, 2009, pp. 1547-1549. doi:10.1253/circj.CJ-08-0269

[4] P. Hoffstetter, B. Djavidani, S. Feuerbach, F. Hofstadter and J. Seitz, "Myxoid Fibrosarcoma of a Pulmonary Vein with Extension into the Left Atrium,” American Journal of Roentgenology, Vol. 186, No. 2, 2006, pp. 365-367. doi:10.2214/AJR.04.1548

[5] P. A. Araoz, H. E. Eklund, T. J. Welch and J. F. Breen, "CT and MR Imaging of Primary Cardiac Malignancies," Radiographics, Vol. 19, No. 6, 1999, pp. 1421-1434.

[6] A. Mingoli, A. Cavallaro, P. Sapienza, L. di Marzo and R. J. Feldhaus, "International Registry of Inferior Vena Cava Leiomyosarcoma: Analysis of a World Series on 218 Patients,” Anticancer Research, Vol. 16, No. 5B, 1996, pp. 3201-3206.

[7] A. Pervaiz, N. Colterjohn, F. Farrokhyar, R. Tozer, A. Figueredo and M. Ghert, “A Systematic Meta-Analysis of Randomized Controlled Trials of Adjuvant Chemotheapy for Localized Resectable Soft-Tissue Sarcoma,” Cancer, Vol. 113, No. 3, 2008, pp. 573-581. doi:10.1002/cncr.23592

[8] A. P. Burke and R. Virmani, "Sarcomas of the Great Vessels: A Clinicopathologic Study," Cancer, Vol. 71, No. 5, 1993, pp. 1761-1773.

doi:10.1002/1097-0142(19930301)71:5<1761::AID-CNC R2820710510>3.0.CO;2-7

[9] B. R. Oliai, H. D. Tazelaar, R. V. Lloyd, M. I. Doria and V. F. Trastek, "Leiomyosarcoma of the Pulmonary Veins," The American Journal of Surgical Pathology, Vol. 23, No. 9, 1999, pp. 1082-1088.

[10] F. Mayer, J. Hartman, et al., "Primary Malignant Sarcomas of the Heart and Great Vessels in Adult Patients-A Single Center Experience,” The Oncologist, Vol. 12, No. 9, 2007, pp. 1134-1142. doi:10.1634/theoncologist.12-9-1134 